\title{
A Spitzer study of interacting luminous and ultra-luminous infrared galaxies
}

\author{
João Rodrigo S. Leão ${ }^{1}$ and Claus Leitherer ${ }^{2}$ \\ ${ }^{1}$ Universidade Federal do Rio Grande - FURG, \\ Instituto de Matemática, Estatística e Fśica - IMEF \\ Caixa Postal 474, Campus Carreiros, Rio Grande, RS, Brazil \\ email: joaoleao@furg.br \\ ${ }^{2}$ Space Telescope Science Institute \\ 3700 San Martin Drive, Baltimore, MD, USA \\ email: leitherer@stsci.edu
}

\begin{abstract}
We conducted a Spitzer Space Telescope survey of 28 Luminous $\left(11<\log \left(L_{I R} / L_{\odot}\right)<\right.$ 12 , LIRGs) and Ultra-Luminous Infrared Galaxies $\left(\log \left(L_{I R} / L_{\odot}\right)>12\right.$, ULIRGs). Many of these galaxies are found in pairs or associations and are powered by either nuclear activity or starformation (Sanders \& Mirabel 1996). Our main goal is to understand the relative importance of starbursts and AGNs in interacting systems. Is the frequency of AGN and starbursts in these interacting galaxies related to their luminosities? What is the importance of the merger stage and the frequency of AGNs? We present our conclusions and diagnostic diagrams based in the observed near infrared lines and compare to studies based solely in optical data.
\end{abstract}

Keywords. techniques: spectroscopy, galaxies: starburst, galaxies: statistics, line: identification, galaxies: interactions

\section{Introduction and Goals}

We present the results of a Spitzer Space Telescope survey of 28 Luminous and UltraLuminous Infrared Galaxies (Leão \& Leitherer 2005, Leão \& Leitherer 2008). The observations were performed using the IRS spectrograph in low-resolution to cover the $4-35 \mu \mathrm{m}$ wavelength range. We measured the flux and equivalent widths of the several observed lines. We are particularly interested in emission lines which characterize the presence of AGNs or starbursts as will be discussed in section 2. Some of the observed SEDs are shown in figure 1. In this study the LIRGs were chosen from the study of Arribas et al. (2004) and images from the Nordic Optical telescope (NOT) are available for these galaxies. The ULIRGs were chosen from the study of Bushouse et al. (2002) and Hubble Space Telescope (HST) images are available. Many of these galaxies are found in pairs or associations (e.g., Bushouse et al. 2002, Arribas et al. 2004) and are believed to be powered by a combination of nuclear activity and star-formation (e.g., Genzel \& Cesarsky 2000, Sanders \& Mirabel 1996).

The study of Sanders et al. (1988) suggests that the fraction of AGNs inreases with the luminosity of the host galaxy. In fact, the optical study of Kim, Veilleux \& Sanders (1998), hereafter KVS98, indicates that for LIRGs, AGNs are present in about $32 \%$ of their galaxies, while for ULIRGs, AGNs (Sey. 1, 2 and LINERs) dominate and are present in $70 \%$ of the systems. In many cases AGNs and starbursts coexist, making it even harder to understand the relative importance of nuclear activity and star-formation in LIRGs and ULIRGs.

The main goal of this project in to understand if diagnostics based in the near infrared spectra yield the same results as KVS98. Do we find the same relative frequency of 
starbursts and AGNs as a function of luminosity? What is the importance of the merger stage and the frequency of AGNs?

\section{Diagnostic Diagrams}

We used the observed nearIR spectra to determine the activity class of our galaxies independently of their optical classification. The presence of the PAH lines at 6.2, 7.7, and $11.3 \mu \mathrm{m}$ are strong indications for the presence of starbursts since the PAH molecules are destroyed (or hidden) by nuclear activity (AGN). The presence of the $[\mathrm{Ne} \mathrm{V}]$ line at 14.3 and $24.3 \mu \mathrm{m}$ is a strong evidence for an AGN since it takes $97 \mathrm{eV}$ to ionize [NeIV] and this energy cannot come from OB stars. The AGNs also exhibit a featurefree continuum below $5 \mu \mathrm{m}$ that cannot be found in starbursts.

In figure 2 we investigate the strength of the $7.7 \mathrm{PAH}$ emission line vs. the $[\mathrm{NeV}]$ $14.3 \mu \mathrm{m}$ emission line and find that starbursts and AGN's are clearly separated. We designed other diagnostic diagrams using the [OIV] at $25.9 \mu \mathrm{m}$ and [NeII] at $12.8 \mu \mathrm{m}$. All these diagrams successfully separate galaxies with AGN activity from those powered by starbursts.

\section{Results}

Our main findings are:(i) Most AGNs are also advanced mergers. This is in agreement with the Sanders et al. (1988) scenario to explain LIRGs and ULIRGs; (ii) We find that for $11<\log \left(L_{I R} / L_{\odot}<12\right.$ (LIRGs) starbursts dominate with $75 \%$ (25\% AGNs), while

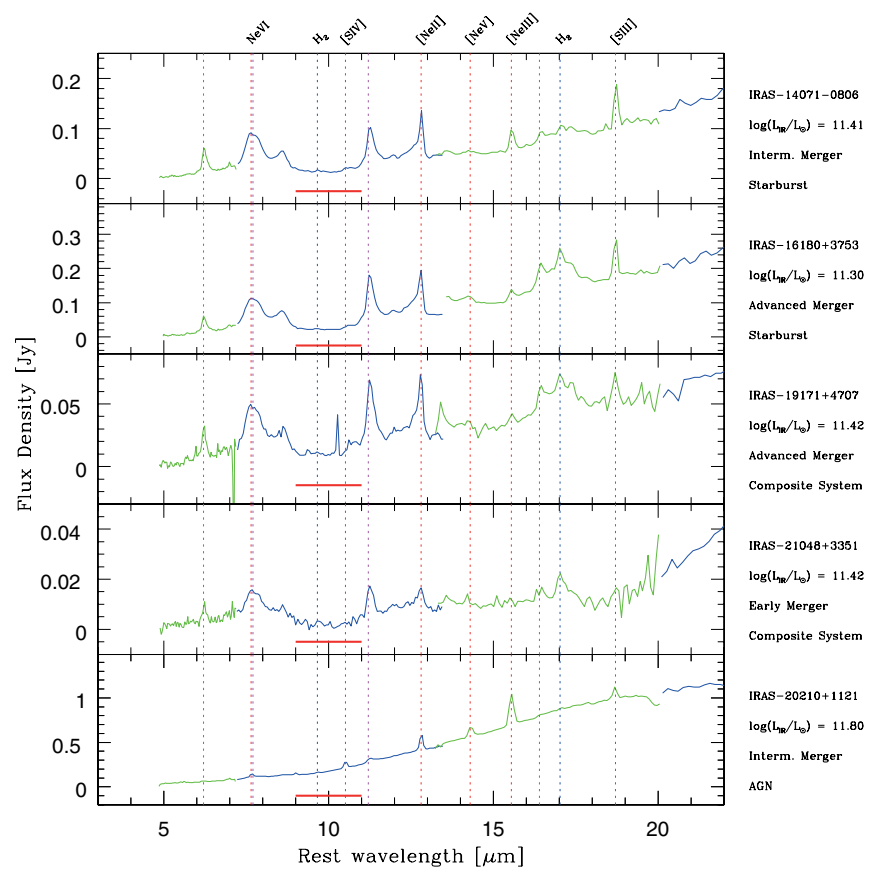

Figure 1. The IRS/SPitzer lowresolution spectra showing in detail the region between $4-22 \mu \mathrm{m}$ for the indicated galaxies, representative of the whole sample. Infrared luminosities, merger stages and activity class are also indicated. The main emission lines are indicated by vertical dotted lines: PAH lines, forbidden emission lines and molecular Hidrogen. The horizontal bar indicates the extent of the silicate absorption around $10 \mu \mathrm{m}$. 


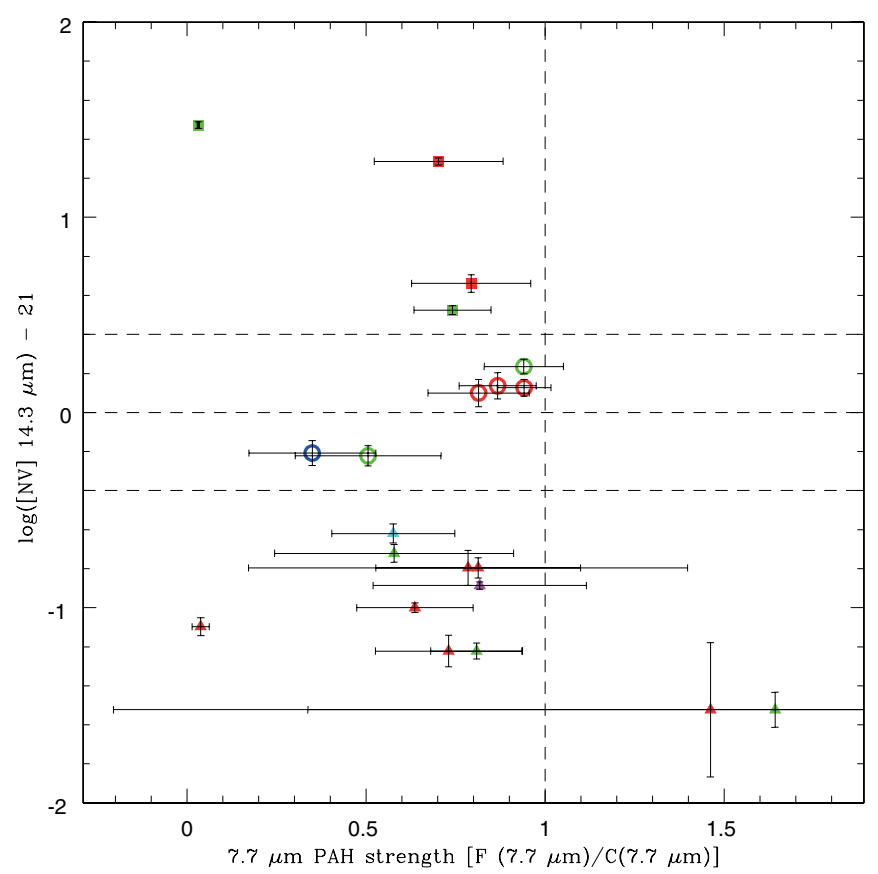

Figure 2. In this plot triangles denote starbursts, circles composite systems and squares AGNs. We see that AGNs and composite systems have stronger [N V] emission lines. Strong $7.7 \mu \mathrm{m}$ PAH lines are seen in AGN's, composites and starbursts, possibly indicating that star formation and nuclear activity might coexist in a single galaxy.

for $\log \left(L_{I R} / L_{\odot}\right) \geqslant 12$ (ULIRGs), AGNs and composite systems dominate with $92 \%$. We thus find a strong evidence for the so called luminosity dependence; (iii) Based on this small sample we find no evidence that advanced mergers are preferentially found in more luminous systems. In other words, we cannot claim that the infrared luminosity is a precise tracer of merger activity. Maybe a larger sample can shed some light on this issue. Many of these galaxies show a deeep silicate absorption and this indicates a large optical depth to the nucleus. We thus suspect that the AGN contribution for many of the galaxies in the sample may be larger. This would imply that AGNs remain hidden even at mid-IR wavelengths.

\section{References}

Sanders, D. B., Mirabel, I. F. 1996, ARAA, 34, 749

Leão, J. R. S. \& Leitherer, C. 2005, General Obs. Proposal 20589, Spitzer Space Telescope Leão, J. R. S. \& Leitherer, C., 2008, General Obs. Proposal 20589, Final Report, Spitzer Space Telescope

Bushouse, H. et al. ApJ, 138, 1

Arribas, S., Bushouse, H., \& Lucas, R. A. 2004, ApJ, 127, 2522

Genzel, R. \& Cesarsky, C. 2000, ARAA, 38, 76

Sanders, D. B., Soifer, B. T., Elias, J. H., Neugebauer, G., \& Matthews, K. 1988, ApJ, 328, L35

Kim, D. C., Veilleux, S., \& Sanders, D. B. 1998, ApJ, 508, 627 